\title{
The value of Contrast-Enhanced Ultrasound in the characterization of vascular pattern of solid pancreatic lesions
}

\author{
Melania Ardelean ${ }^{1}$, Roxana Sirli' ${ }^{1}$ Ioan Sporea ${ }^{1}$, Simona Bota ${ }^{1}$, Mirela Dănilă ${ }^{1}$, \\ Alina Popescu' ${ }^{1}$ Bogdan Timar², Roxana Buzas ${ }^{3}$, Octavian Mazilu³, Ovidiu Ardelean³, \\ Daniel Lighezan ${ }^{3}$
}

${ }^{1}$ Department of Gastroenterology and Hepatology, "Victor Babeș" University of Medicine and Pharmacy, ${ }^{2}$ Department of Informatics and Biostatistics, "Victor Babes" University of Medicine and Pharmacy, ${ }^{3}$ Municipal County Hospital, "Victor Babes" University of Medicine and Pharmacy, Timisoara, Romania

\begin{abstract}
The aim of our study was to evaluate the accuracy of CEUS in the characterization of pancreatic solid lesions, considering cross sectional imaging techniques (CE-CT/MRI) as the "gold standard" methods. Material and methods: We performed a retrospective, monocentric study that included 91 solid pancreatic lesions which were evaluated by CEUS and by a secondline contrast imaging technique (CT or MRI), considered as the reference method. Results: The rate of a conclusive diagnosis based on a typical enhancement pattern was $94 \%$ ( $78 / 83$ cases). In 72 cases out of $83(86.7 \%)$ there was a perfect concordance between CEUS and the "gold-standard" imaging method (CE-CT/MRI). In our study, 88\% (73/83) of the pancreatic lesions were categorized as malignant due to their typical wash-out aspect in the late phase. The overall accuracy of CEUS for the differential diagnosis of solid pancreatic tumors was approximately $81 \%$. The accuracy of CEUS for the diagnosis of hypoenhancing pancreatic tumors was approximately $89.1 \%$; while for the diagnosis of hyperenhancing pancreatic tumors it was approximately $72.8 \%$. Conclusion: CEUS allows the differentiation between hypo- vs. hyperenhancing pancreatic solid lesions, with a considerable diagnostic accuracy, a fundamental step in the precise diagnosis of pancreatic tumors.

Keywords: CEUS, enhancement pattern, pancreatic tumors, cross sectional imaging techniques
\end{abstract}

\section{Introduction}

More and more pancreatic lesions (symptomatic or not) are being detected due to recent advancement of imaging technology. Differentiation between various types of pancreatic tumors is crucial for the patients' prognostic. Since surgical resection offers the best chance for long-term survival and in order to avoid unnecessary laparotomies, the characterization of pancreatic masses based on imaging modalities is mandatory, but still challenging [1].

Conventional B-mode ultrasound (US) is a preferred imaging method in the screening for pancreatic lesions [2] because of its relatively low cost, readily available exami-

Received 17.12.2014 Accepted 15.01.2015

Med Ultrason

2015, Vol. 17, No 1, 16-21

Corresponding author: Roxana Şirli

14 , Sirius str, ap. 5 ,

300688 Timisoara, Romania

E-mail address: roxanasirli@gmail.com nation [3] and, not the least, because it is a non-invasive method. Until now, due to its accessibility and its ability to perform a rapid scan of the whole abdomen and pelvis, the main diagnostic method for pancreatic lesions had been contrast enhanced - computer tomography (CECT). Magnetic resonance imaging (MRI), which showed a higher specificity and sensitivity in the detection and staging different types of pancreatic lesions, is more expensive and not so widely available. The introduction of contrast-enhanced ultrasound (CEUS) has improved the diagnostic accuracy of US and extended its value $[3,4]$. The innovative use of CEUS for the study of the pancreas created the need for a precise definition of the most dynamic features of pancreatic masses (solid and cystic) [5]. Thus, CEUS can aid in the differential diagnosis of pancreatic tumors [4] and can provide dynamic information, with a high temporal and spatial resolution, concerning macro and micro-circulation of focal lesions and normal parenchyma $[3,6]$. Its high capability in showing tumoral microcirculation also makes CEUS accurate in the study 
of neoangiogenesis [5,7]. But CEUS has the disadvantage of being an operator-dependent method, and also its accuracy is influenced by the patients' acoustic window, an important aspect to be taken into consideration when evaluating a pancreatic lesion.

Currently, a number of published papers describe the CEUS enhancement pattern of the most common benign and malign pancreatic lesions [3,8-14].

The aim of our study was to evaluate the accuracy of CEUS in the characterization of pancreatic solid lesions, considering CECT/MRI as the "gold standard" methods.

\section{Material and methods}

We performed a retrospective, monocentric study that included 91 solid pancreatic lesions which were evaluated in our Department during a 50 months period, between October 2009 and December 2013.

\section{Patients}

We included in our study patients older than 18 years, in whom focal solid pancreatic masses were found and could not be characterized by US alone. In each patient the following characteristics were documented: indication for CEUS and a short history regarding pancreatic diseases or pancreatic malignancy. Each patient underwent a B-mode US examination, followed in the same session by a CEUS examination. CE-CT or MRI exam were available in each patient and considered as the "gold standard" for establishing the final diagnosis.

Exclusion criteria were: absence of the gold standard method (CE-CT/MRI); a characterized pancreatic lesion before the present study; inconclusive aspect in CE-CT/ MRI; difficulties in CEUS examination due to the lesion' location (tail of the pancreas, improper acoustic window); subjects with a recent myocardial infarction; class III/IV cardiac insufficiency; significant rhythm disorders; pregnant women.

The study was conducted in full accordance with the Declaration of Human Rights (Helsinki, 1975) and with its further revisions (2000 revision-Edinburgh), and was approved by the local ethical committee. Patients gave their written consent prior examination.

\section{Methods}

All the studied lesions respected the same approach: standard US and CEUS in the same session, followed by CT/MRI. CEUS examinations were performed in a highly experienced center, by 4 experienced physicians, $2^{\text {nd }}$ and $3^{\text {rd }}$ level according to the European Federation of Societies for Ultrasound in Medicine and Biology (EFSUMB) classification of expertise levels. CEUS was performed on an Accuson S2000 US system (Siemens, Er-

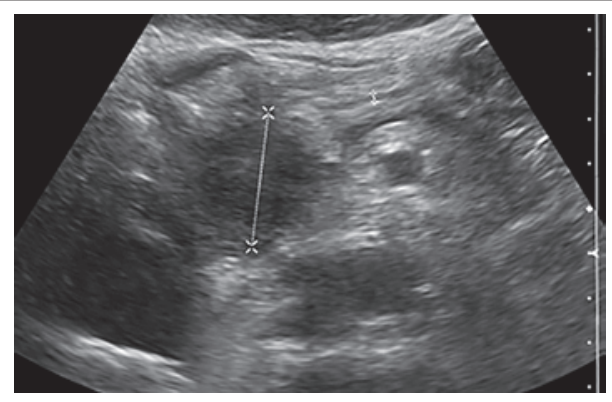

Fig 1. Hypoechoic lesion in the pancreatic head. Dilated Wirsung duct (B-mode ultrasound).

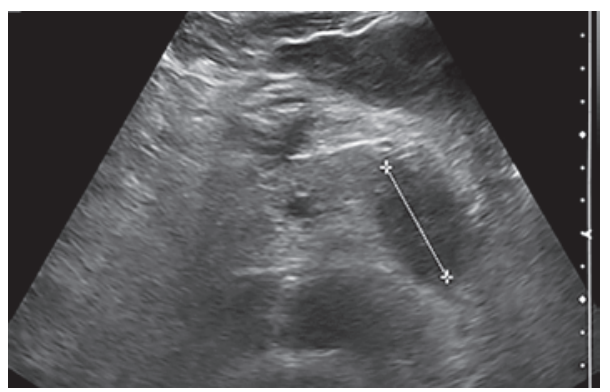

Fig 2. Hypoechoic lesion in the pancreatic tail (B-mode ultrasound).

langen, Germany) with a low mechanical index: 0.09 to 0.11 . Firstly a standard US examination was performed and the pancreatic lesions' size, location and pattern were documented (fig 1, fig 2). Afterwards, a CEUS was performed in the same session only for the assessment of "de novo" solid pancreatic masses (undetermined new pancreatic mass, not characterized by another imaging method). We did not include into the study cystic lesions (anechoic in B-mode ultrasound); mixed, solid-anechoic lesions; nor the lesions (solid and/or cystic) detected during an acute pancreatitis.

Real-time observation of the lesion's blood perfusion was no less than 120 s, during which the patient was instructed to maintain smooth breathing. Dynamic images were preserved for later analysis. A $2.4 \mathrm{ml}$ bolus of second generation contrast agent - sulphur hexafluoride filled microbubbles with a phospholipid peripheral shell (SonoVue, Bracco SpA, Milan, Italy) - was injected in an antecubital vein, followed by a 10 $\mathrm{ml}$ bolus saline solution. A "real-time", dynamic observation of the contrast enhanced phases - arterial (early stage of enhancement, until 30 seconds) and late (delayed stage of enhancement, 30-45 until 120 seconds following contrast injection) - began immediately after the contrast bolus $[2,3,15]$. Considering the surrounding pancreatic parenchyma as control, the enhancement and wash-out of the contrast agent in the lesion was observed. 
Table I. Criteria used for pancreatic tumor differentiation in US [6,18] and CEUS [16]:

\begin{tabular}{lll}
\hline Pancreatic lesion & B-mode ultrasound & CEUS \\
\hline Ductal carcinoma & Low echo pattern & Typically hypoenhanced \\
& Lobulated margins \\
& Dilated Wirsung's duct & \\
Pancreatitis (chronic, autoimmune) & Low- echo pattern & Autoimmune pancreatitis presents as isoenhanced; chronic \\
& Lobulated margins & pancreatitis as isoenhanced. \\
& Thrombosis & \\
& Dilated Wirsung's duct & \\
& Calcifications & \\
& Low echo pattern & Typically hyperenhanced \\
& Sharply delineated & \\
& Wirsung duct no-dilated & \\
\hline
\end{tabular}

According to the enhancement pattern as compared to the surrounding pancreas, the lesion was categorized as hyper-, hypo- or isoenhancing: 1) Homogenous hyperenhancement: the whole lesion enhanced homogenously

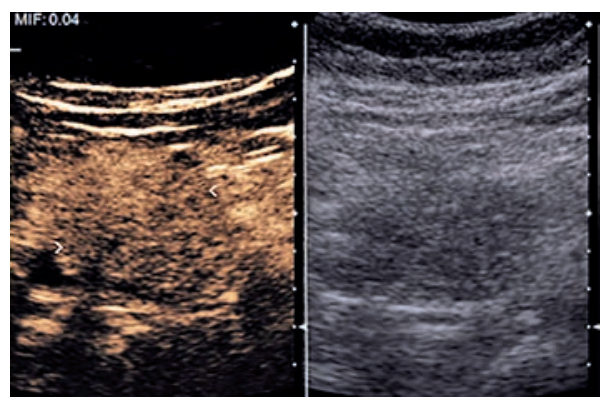

Fig 3. Hypoenhanced lesion in the pancreatic body - arterial phase (arrows).

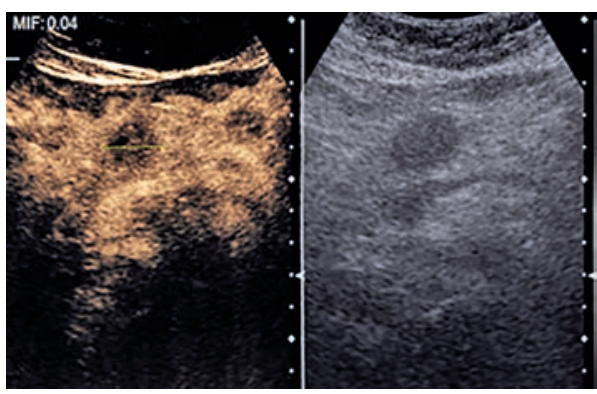

Fig 4. Hypoenhanced lesion in the pancreatic body - arterial phase.

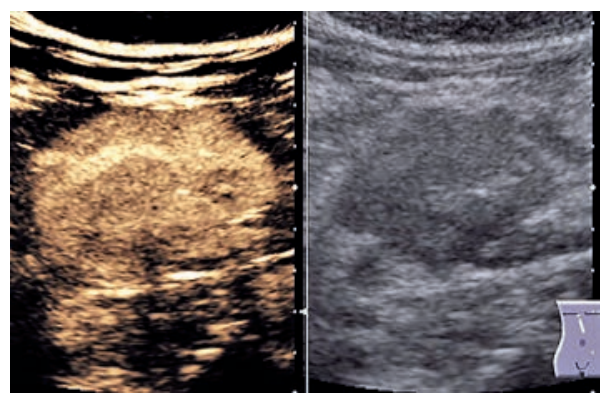

Fig 5. Hyperenhanced lesion in the pancreatic body - arterial phase following the contrast bolus; 2) Heterogeneous hyperenhancement: the pancreatic lesion enhanced inhomogenously following the contrast bolus; 3) Isoenhanced: the lesion's enhancement was similar to the surrounding pancreatic parenchyma; 4) Hypoenhancement: the lesion enhanced less than the surrounding parenchyma; 5) Wash-out: loss of enhancement in the late phase, preceded by hypo-, hyper- or isoenhancement in the arterial phase (fig 3-5).

A CEUS final diagnosis was established after the contrast agent completed its course by comparing the enhancement pattern with the typical one described in the EFSUMB guidelines from 2008, updated in 2011 [16,17]. We compared the results of the enhancement pattern in our study with a second line contrast imaging method (CT/MRI), considered to be the reference method, and identified the accuracy of CEUS in the characterization of solid pancreatic masses.

The criteria used for pancreatic tumor differentiation in US $[6,18]$ and CEUS [16], are presented in Table I.

\section{Statistical analysis}

Data were collected and analyzed using the SPSS v.17 software suite (SPSS Inc. Chicago, IL, USA). Data are presented as mean \pm standard deviations for continuous variables with Gaussian distribution. For analyzing the diagnosis quality of pancreatic CEUS we used sensitivity ( $\mathrm{Se}$ - the number of true positive divided to the total number of positives), specificity ( $\mathrm{Sp}-$ the number of true negatives divided to the total number of negatives), positive predictive value (PPV - the number of true positives divided to the total number of positives at test) and negative predictive value (NPV - the number of true negatives divided tot the total number of negatives at the test). Accuracy was defined as the percentage of correctly classified patients from the total analyzed ones. A value of $p<0.05$ was considered statistically significant and was taken into consideration. 
Table II. Patients' characteristics

\begin{tabular}{ll}
\hline Median age (years) & $63.7 \pm 9.8$ \\
\hline Gender & \\
- women & $49(59 \%)$ \\
- men & $34(41 \%)$ \\
\hline Reference Method (CT/MRI) & $67(80.7 \%)$ cases with CT \\
& $16(19.3 \%)$ cases with MRI \\
\hline CEUS diagnosis: & \\
- hypoenhanced tumors & $39(47 \%)$ \\
- hyperenhanced tumors & $31(37.3 \%)$ \\
- isoenhanced tumors & $13(15.7 \%)$ \\
\hline Final diagnosis by CT/MRI: & \\
- hypoenhanced tumors & $36(43.3 \%)$ \\
- hyperenhanced tumors & $28(33.7 \%)$ \\
- isoenhanced tumors & $12(14.5 \%)$ \\
- homogenous pancreatic tissue & $2(2.4 \%)$ \\
- solid-cystic tumors & $5(6.1 \%)$ \\
\hline
\end{tabular}

\section{Results}

We investigated 91 CEUS examinations in 91 patients. Of the whole investigated group we excluded 8 cases ( 5 cases in which the gold-standard method, CECT/MRI, was not available and 3 cases in which the lesion, located in the tail of the pancreas, was not clearly seen due to a poor ultrasound window). Thus, 83 lesions in 83 patients were included in the final analysis. The patients' characteristics are presented in Table II.

The pancreatic lesions' aspect in standard US is detailed in Table III.

The rate of a conclusive diagnosis based on a typical enhancement pattern was $94 \%$ ( $78 / 83$ cases). In 72 cases out of $83(86.7 \%)$ there was a perfect concordance between CEUS and the "gold-standard" imaging method (CE-CT/MRI). In our study, 88\% (73/83) of the pancre- atic lesions were categorized as malignant due to their typical wash-out aspect in the late phase.

The performance of CEUS for the differential diagnosis of solid pancreatic tumors is presented in table IV.

\section{Discussions}

The main imaging methods used for the assessment of pancreatic lesions are US, CE-CT or MRI. Conventional US is the preferred first-line method since it is noninvasive, simple and inexpensive. However, its quantitative diagnostic ability is limited [2]. Major limitations are the occasionally restricted image resolution due to the lesions' location (especially in the pancreatic tail) and to the poor sonographic visualization of the gland, due to overlying abdominal gas or to large amounts of abdominal fat [5].

In the last years, CEUS became a validated imaging technique for the assessment of pancreatic pathology. The main advantage of CEUS, as compared to other imaging techniques, is the ability to obtain a dynamic study, a real-time observation of blood perfusion of the lesion. With high spatial and temporal resolution for a relatively long time after microbubbles bolus, CEUS tends to gain lot of confidence in the characterization of pancreatic lesions $[2,19]$. Due to the fixed time of scanning, CE-CT/ MRI cannot capture a transient enhancement. Besides, it is difficult for CE-CT/MRI to reflect the full-time phase blood supply of tumor [2].

When a pancreatic tumor has been detected, an immediate and correct differential diagnosis is mandatory to direct appropriate management [3]. CEUS is able to provide a rapid assessment of the pancreatic lesion's pat-

Table III. Pancreatic lesions' characteristics in standard ultrasonography (83 lesions).

\begin{tabular}{lll}
\hline Size n (\%) & Location n (\%) & Aspect in US n (\%) \\
\hline$<2 \mathrm{~cm}: 3(3.6 \%)$ & Head: $53(63.8 \%)$ & Hyperechoic: $4(4.8 \%)$ \\
$2-5 \mathrm{~cm}: 59(71.1 \%)$ & Isthm: $12(14.5 \%)$ & Hypoechoic: $47(56.6 \%)$ \\
$>5 \mathrm{~cm}: 9(10.8 \%)$ & Body: $7(8.5 \%)$ & Hypo-hyperechoic: $3(3.6 \%)$ \\
Not recorded: $12(14.5 \%)$ & Body-Tail: $4(4.8 \%)$ & Mixed: $3(3.6 \%)$ \\
& Tail: $1(1.2 \%)$ & Hypo-anechoic: $5(6.1 \%)$ \\
& Procesus uncinatus: $4(4.8 \%)$ & Isoechoic: $17(20.5 \%)$ \\
& Not recorded: $2(2.4 \%)$ & Not recorded: $4(4.8 \%)$ \\
\hline
\end{tabular}

$\mathrm{n}$ - number of lesions

Table IV. Performance of CEUS for the differential diagnosis of solid pancreatic tumors

\begin{tabular}{lllllll}
\hline & \multicolumn{3}{l}{ Overall (all tumors) } & \multicolumn{2}{l}{ Hypoenhancing tumors } & \multicolumn{2}{l}{ Hyperenhancing tumors } \\
& \% & $\mathbf{9 5 \%}$ CI & \% & $\mathbf{9 5 \%}$ CI & \% & 95\% CI \\
\hline Sensitivity & 90.7 & 85.3 to 96.2 & 92.2 & 87.9 to 96.6 & 79.8 & 65.7 to 93.9 \\
Specificity & 90 & 84.6 to 95.5 & 86.4 & 78.1 to 94.7 & 97.8 & 95.9 to 99.8 \\
Positive Predictive Value & 87.2 & 80.4 to 94.1 & 88.7 & 81.8 to 95.7 & 75.7 & 51.7 to 99.7 \\
Negative Predictive Value & 95.4 & 91.5 to 99.3 & 94.2 & 88.8 to 99.6 & 96.5 & 93.6 to 99.5 \\
\hline
\end{tabular}

CI- confidence interval 
tern and to improve the solid lesion characterization, thus making possible a differential diagnosis. The most common solid pancreatic tumors are adenocarcinomas. Neuroendocrine lesions and metastases in the pancreas occur less frequently [18].

The overall accuracy of CEUS for the differential diagnosis in solid pancreatic masses was $81 \%$ in our study, a result slightly lower than in the Pancreatic Multicenter Ultrasound Study (PAMUS) in which the overall accuracy was $91.7 \%$ [3]. Previous studies report CEUS accuracies for the diagnosis of pancreatic solid lesions ranging from $83 \%$ to $92 \%$ [20,21]. The overall accuracy might be higher in PAMUS since pancreatitis lesions were also included in the group of solid pancreatic lesions, with a strong accuracy of $96.7 \%$. In our study we only included only solid pancreatic tumors and excluded patients with pancreatitis.

The diagnostic accuracy of CEUS in hypoenhancing pancreatic lesions was $89.1 \%$ in our study, which was consistent with the results of studies by Yan et al and D'Onofrio et al $[19,20]$. In particular, in PAMUS [3] ductal adenocarcinomas were correctly identified with an accuracy of $87.8 \%$ based on the hypoenhancing aspect at CEUS. In five series that included more than fifty ductal adenocarcinomas proven by pathological exam, the tumor was reported to be hypovascular in $73-93 \%$ of the cases [10-12,16,22]. Our results are slightly better, possibly due to the fact that the gold standard method in our study was an imaging technique, not pathology.

In our study, 3 lesions described by CEUS with hypoenhancing pattern had a discordant aspect in the gold standard method: hyperenhancing (2 lesions) and isoenhancing (1 lesion). An explanation may be the presence of distorted tumor vessels, of abnormal structures and vascular fistula caused by carcinoma invasion, so that the contrast agent has an inhomogeneous distribution, with a rapid wash-out [2].

Even if the accuracy of CEUS in characterizing hypovascular pancreatic lesions is high when compared with CE-CT/MRI, CEUS alone is not enough for a correct tumor staging. To assess tumor resectability, contrast enhanced CT/MRI are needed for a more accurate evaluation of the local extension and metastatic spread [6], even if in the same CEUS session we can examine the liver in the late phase to search for metastases. Thus, the most important role of CE-CT/MRI is to search for a possible invasion of the adjacent vessels.

CEUS also seems very accurate in detecting the hyperenhancing pattern of a pancreatic lesion. In the present study, the accuracy of CEUS in characterizing hyperenhancing lesions was $72.8 \%$, similar to the one published by Serra et al [23] $-73.8 \%$; but slightly lower than in the PAMUS, in which the accuracy was $90.5 \%$ [3]. This might be due to the small number of hyperenhancing lesions identified by CE-CT/MRI ( 28 cases) and also because we couldn't verify the imaging results with a more sensitive method (pathology). In PAMUS, most of the neuroendocrine tumors had a hyperenhancing pattern, but some lesions also presented a hypoenhancing or isoenhancing pattern, depending on the amount of stroma within a tick and hyalinized lesion. In our study 3 lesions interpreted by CEUS as hyperenhancing, were described as hypoenhancing in CE-CT/MRI, a situation also described in PAMUS [3].

By combining both methods (CEUS and CE-CT/ MRI), a higher accuracy in detecting a neuroendocrine tumor, or in characterizing the extent of an adenocarcinoma can be expected. As stated in PAMUS, CEUS can improve the accuracy of the ultrasound study of pancreatic masses, obtaining faster diagnosis (immediate diagnosis of ductal adenocarcinoma). CEUS could also play a further role, subject for additional investigations, and act as a problem solving method: uncertain hypervascularity, or doubtful septa enhancement in a lesion [3]. Here is the place where the second imaging method and or histology interfere.

In the isoenhancing group, 7 lesions turned to be chronic pancreatitis, even though in CEUS they presented a delayed wash-out and 2 lesions were autoimmune pancreatitis. The other 3 lesions were interpreted by CE$\mathrm{CT} / \mathrm{MRI}$ as isoenhancing tumors with rapid wash-out.

A major limitation of the present study is that the reference method was not the pathology but an imaging technique. That's why we defined each lesion as being hypo, hyper or isoenhancing and why we couldn't establish a firm diagnosis (adenocarcinoma, neuroendocrine tumor). The reference method, CE-CT/MRI, also described the lesions' enhancement pattern, and not the histological diagnosis. Nevertheless, our results were similar to the ones presented in published studies, where histology was considered the "gold standard" method.

Others limitations of the present study are the operator-dependency of US/CEUS and the fact that CE-CT/ MRI were performed in different centers and interpreted by different examiners.

\section{Conclusions}

CEUS allows with a considerable diagnostic accuracy the differentiation between hypo- vs. hyperenhancing pancreatic solid lesions, which is a fundamental step in the precise diagnosis of pancreatic tumors. Thus, CEUS proved to be highly concordant (almost $87 \%$ ) with a second-line cross sectional imaging method for the diagno- 
sis of pancreatic solid lesions, proving its high accuracy of almost $81 \%$.

Acknowledgement: The research published in this paper was made with support from the grant awarded by the "Victor Babes" University of Medicine and Pharmacy Timisoara, in PROGRAMUL III - C2 - PCFI 2015/2016.

\section{Conflict of interest: none}

\section{References:}

1. Tawada K, Yamaguchi T, Kobayashi A, et al. Changes in tumor vascularity depicted by contrast-enhanced ultrasonography as a predictor of chemotherapeutic effect in patients with unresectable pancreatic cancer. Pancreas 2009; 38: 3035.

2. Fan Z, Li Y, Yan K, et al. Application of contrast enhancedultrasound in the diagnosis of solid pancreatic lesions- A comparison of conventional ultrasound and contrast-enhanced CT. Eur J Radiol 2013; 82: 1385-1390.

3. D'Onofrio M, Barbi E, Dietrich CF, et al. Pancreatic multicenter ultrasound study (PAMUS). Eur J Radiology 2012; 81: 630-638.

4. Kersting S, Roth J, Bunk A. Transabdominal contrast-enhanced ultrasonography of pancreatic cancer. Pancreatology 2011; 11 Suppl 2: 20-27.

5. D’Onofrio M, Galloti A, Principe F, Mucelli RP. Contrastenhanced ultrasound of the pancreas. World J Radiol 2010; 2: $97-102$.

6. Recaldini C, Carrafiello G, Bertolotti E, Angeretti MG, Fugazzola C. Contrast-enhanced ultrasonograpic findings in pancreatic tumors. Int J Med Sci 2008; 5: 203-208.

7. D’Onofrio M, Malago R, Zamboni G, et al. Contrast-enhanced ultrasonography better identifies pancreatic tumor vascularization than helical CT. Pancreatology 2005; 5: 398-402.

8. Numata K, Ozawa Y, Kobayashi N, et al. Contrast-enhanced sonography at pancreatic carcinoma: correlation with pathological findings. J Gastroenterology 2005; 40: 631-640.

9. Dietrich CF, Braden B, Hocke M, Ott M, Ignee A. Improved characterization of solitary solid pancreatic tumors using contrast enhanced transabdominal ultrasound. J Cancer Res Clin Oncol 2008; 134: 635-643.
10. Kitano M, Kudo M, Maekawa K, et al. Dynamic imaging of pancreatic diseases by contrast-enhanced coded phase inversion harmonic ultrasonography. Gut 2004; 53: 854-859.

11. Sofuni A, Iijima H, Moriyasu F, et al. Differential diagnosis of pancreatic tumors using ultrasound contrast imaging. J Gastroenterology 2005; 40: 518-525.

12. Nagase M, Furuse J, Ishii H, Yoshino M. Evaluation of contrast enhanced patterns in pancreatic tumors by coded harmonic sonographic imaging with a microbubble contrast agent. J Ultrasound Med 2003; 22: 789-795.

13. D'Onofrio M, Zamboni G, Tognolini A, et al. Mass-forming pancreatitis: value of contrast-enhanced ultrasonography. World J Gastroenterol 2006; 12: 4181-4184.

14. Rickes S, Rauh P, Uhle C, Ensberg D, Mönkemüller K, Malfertheiner P. Contrast-enhanced sonography in pancreatic diseases. Eur J Radiol 2007; 64: 183-188.

15. D'Onofrio M, Canestrini S, Crosara S, De Robertis R, Pozzi Mucelli R. Contrast enhanced ultrasound with quantitative perfusion analysis for objective characterization of pancreatic ductal adenocarcinoma: A feasibility study. World J Radiol 2014; 6: 31-35.

16. Piscaglia F, Nolsoe C, Dietrich CF, et al. The EFSUMB Guidelines and Reccomendations on the Clinical Practice of Contrast Enhanced Ultrasound (CEUS): update 2011 on non-hepatic applications. Ultraschall Med 2012; 33: 33-59.

17. Claudon M, Cosgrove D, Albrecht T, et al. Guidelines and good clinical practice recommendations for contrast enhanced ultrasound (CEUS)-update 2008. Ultraschall Med 2008; 29: 28-44.

18. Rickes S, Monkemuller K, Malfertheiner P. Contrast-enhanced ultrasound in the diagnosis of pancreatic tumors. JOP 2006; 7: 584-592.

19. D'Onofrio M, Crosara S, Signorini M, et al. Comparison between CT and CEUS in the diagnosis of pancreatic adenocarcinoma. Ultraschall in Med 2013; 34: 377-381.

20. Yan K, Dai Y, Wang YB, et al. The role of contrast-enhanced ultrasound in diagnosing pancreatic diseases. Chinese Journal of Ultrasonongraphy 2006; 15: 361-364.

21. Xiaoling YU, Ping L, Bao-wei D, et al. The value of contrast enhanced ultrasound in the diagnosis of pancreatic lesions. Chinese Journal of Medical Imaging 2008; 16: 170-173.

22. Hocke M, Schultze E, Gottschalk P, Topalidis T, Dietrich CF. Contrast-enhanced ultrasound in discrimination between focal pancreatitis and pancreatic cancer. World J Gastroenterol 2006; 12: 246-250.

23. Serra C, Felicani C, Mazzotta, et al. Contrast-enhanced ultrasound in the differential diagnosis of exocrine versus neuroendocrine pancreatic tumors. Pancreas 2013; 42: 871-877. 